\title{
Aortic valve replacement and mitral valve repair as treatment of complications after percutaneous core valve implantation
}

\author{
Chiara Comoglio, MD, ${ }^{\text {a }}$ Massimo Boffini, MD, ${ }^{\mathrm{a}}$ Suad El Qarra, MD, ${ }^{\mathrm{a}}$ Fabrizio Sansone, MD, ${ }^{\mathrm{a}}$ \\ Maurizio D'Amico, MD, ${ }^{b}$ Sebastiano Marra, MD, ${ }^{b}$ and Mauro Rinaldi, Professor, ${ }^{a}$ Turin, Italy
}

Standard aortic valve replacement is the gold standard for the treatment of severe aortic stenosis but transcatheter procedure can be performed in selected patients with important comorbidities or absolute contraindication for standard surgical approach. Careful multidisciplinary evaluation of each single case is fundamental in order to tailor the appropriate approach for every patient.

\section{CLINICAL SUMMARY}

We describe the case of a 66-year-old man who had surgical intervention for a core valve malfunction 3 months after transfemoral implantation. The patient was scheduled for percutaneous aortic valve implantation (PAVI) because of the presence of numerous risk factors, such as obesity (body mass index, 35\%) and myelodysplasia. The main concern about elective standard aortic valve replacement was related to the patient's pulmonary function. As a result of a history of smoking and obesity, he had severe chronic pulmonary disease (forced expiratory volume in 1 second, $<35 \%$ ) and obstructive sleep apnea syndrome requiring noninvasive mechanical ventilation.

The patient presented with rapid worsening of dyspnea. An echocardiogram showed a severely calcified aortic stenosis with left ventricular hypertrophy and good ejection fraction $(60 \%)$. For this reason, he was evaluated for aortic valve replacement. Preoperative coronary angiographic analysis revealed normal coronary arteries. Despite a relative low EuroSCORE, PAVI was indicated because of multiple comorbidities and requested by the patient.

PAVI with a core valve bioprosthesis was performed without procedural complications. Only a second balloon dilatation was required for a moderate paraprosthetic aortic regurgitation detected immediately after the procedure, and a mild-to-moderate paraprosthetic aortic regurgitation re-

\footnotetext{
From the Division of Cardiac Surgery, ${ }^{\text {a }}$ University of Turin, San Giovanni Battista Hospital, and the Division of Cardiology, ${ }^{\mathrm{b}}$ San Giovanni Battista Hospital, Turin, Italy.

Received for publication Sept 29, 2008; revisions received Oct 22, 2008; accepted for publication Nov 2, 2008; available ahead of print March 18, 2009.

Address for reprints: Fabrizio Sansone, MD, Division of Cardiac Surgery, University of Turin, San Giovanni Battista Hospital, C.so Bramante 88/90, Turin, Italy (E-mail: fabrisans@katamail.com).

J Thorac Cardiovasc Surg 2009;138:1025-7

$0022-5223 / \$ 36.00$

Copyright (c) 2009 by The American Association for Thoracic Surgery

doi:10.1016/j.jtcvs.2008.11.005
}

mained. The following postprocedural period was complicated by several episodes of sustained ventricular tachycardia and 1 episode of ventricular fibrillation requiring automatic cardiac defibrillator implantation. However, no electrocardiographic abnormalities or cardiac enzyme release were detected. The postprocedural rehabilitation period was characterized by slow functional recovery with many episodes of ventricular tachycardia. One month later, the patient was readmitted to the intensive care unit for progressive biventricular failure and ventricular arrhythmia. Echocardiographic analysis confirmed moderate paraprosthetic aortic regurgitation with preserved left ventricular ejection fraction but with progressive left ventricular dilatation. Cardiac rhythm was restored with lidocaine and amiodarone infusion, but acute respiratory failure occurred, requiring intubation and mechanical ventilation for about 1 week.

Two months later, the patient reported progressive worsening of general conditions, with fever, shivering, and leukocytosis. A single blood culture was positive for corynebacterium. An echocardiogram showed what appeared to be a pseudoaneurysm of the posterior aortic annulus. Aortic regurgitation was stable, but severe mitral regurgitation was detected as a result of anterior leaflet perforation, enhancing the suspicion of acute endocarditis. For this reason, 3 months after the percutaneous procedure, the patient underwent surgical intervention.

After median sternotomy, cardiopulmonary bypass was instituted as usual. After clamping the aorta, cardioplegia was administered in the aortic root without success because of severe aortic regurgitation, and selective cardioplegia directly into the coronary ostia was required. Once the aorta was opened, despite a well-positioned aortic valve prosthesis, we found a bioprosthetic metal support protruding for about $4 \mathrm{~cm}$ in the ascending aorta, causing severe obstruction of the left main trunk ostium (Figure 1), and only small vegetation on the cusps was found, without annular involvement (Figure 1). The proximal part of the support was about $5 \mathrm{~cm}$ into the left ventricular outflow tract, leaning against the anterior mitral leaflet and causing leaflet perforation (Figure 2). A paraprosthetic leak was found at the level of the left and noncoronary cusps, where an important calcification was present. To remove the prosthesis, the aortic root was filled with ice water to crimp the core valve, and after a gentle squeeze of the aorta, the core valve was accurately detached from 

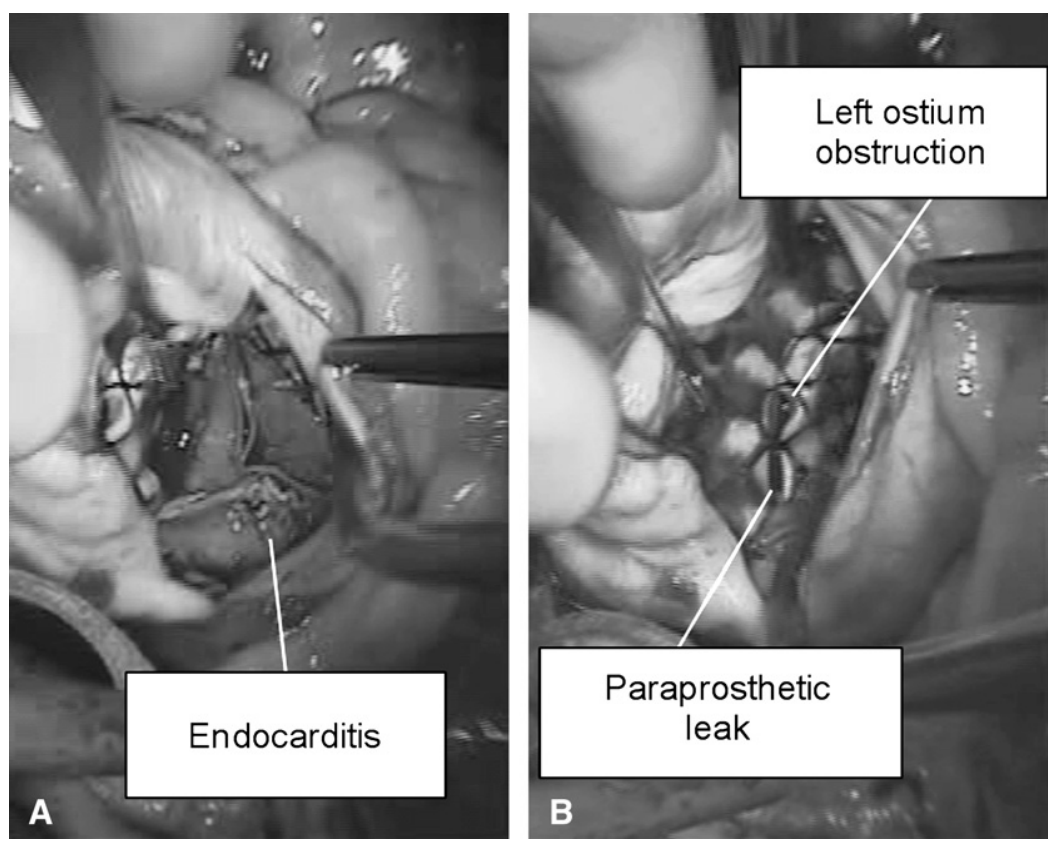

FIGURE 1. A, Prosthetic endocarditis. B, Intraoperative evidence of severe left coronary ostial obstruction caused by core valve metal support and paraprosthetic leak.

the aortic wall itself and successfully removed (Figure 2, B). A 23-mm Carpentier-Edwards aortic valve prosthesis was implanted. Anterior mitral leaflet injury was repaired with a pericardial patch.

The postoperative course was uneventful. The patient was discharged on the seventh postoperative day. Predischarge echocardiographic analysis showed correct function of the aortic bioprosthesis and no residual mitral regurgitation.

\section{DISCUSSION}

Some considerations of our experience are mandatory. First, the design of the core valve is quite bulky, with a part of the prosthesis leaning into the left ventricle with possible interference with the mitral valve apparatus. Moreover, the core valve metal support protrudes into the ascending aorta with the risk of left main obstruction, as in our case.
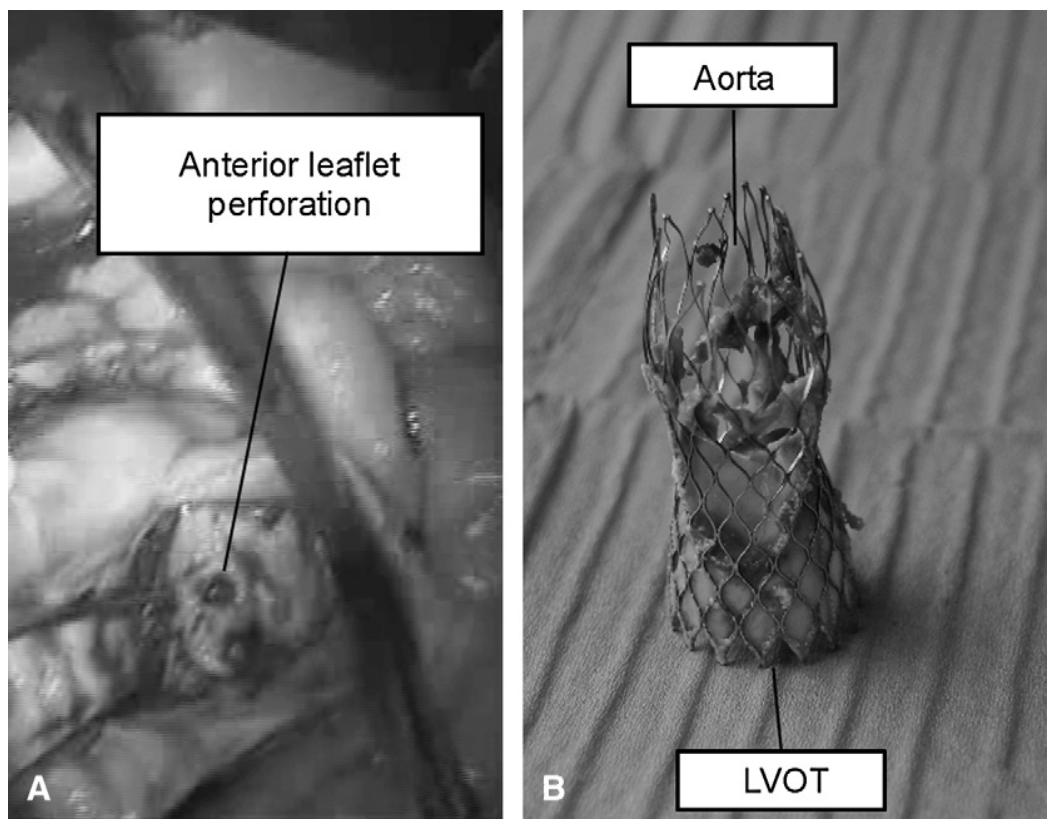

FIGURE 2. A, Anterior mitral leaflet perforation. B, Core valve appearance. LVOT, Left ventricular outflow tract. 
Second, the postoperative course after standard aortic valve replacement was uneventful, despite the important comorbidities of the patient that suggested PAVI. Therefore an accurate preoperative evaluation is fundamental to identify those patients who are not suitable for the standard surgical procedure.

\title{
Totally percutaneous valve replacement for severe aortic regurgitation in a degenerating bioprosthesis
}

\author{
Massimo Napodano, MD, ${ }^{\mathrm{a}}$ Ada Cutolo, MD, ${ }^{\mathrm{a}}$ Chiara Fraccaro, MD,${ }^{\mathrm{a}}$ Giuseppe Tarantini, MD, PhD, ${ }^{\mathrm{a}}$ \\ Raffaele Bonato, MD, ${ }^{\mathrm{b}}$ Roberto Bianco, MD,${ }^{\mathrm{c}}$ Gino Gerosa, $\mathrm{MD},{ }^{\mathrm{c}}$ Sabino Iliceto, ${ }^{\mathrm{a}}$ and \\ Angelo Ramondo, MD, ${ }^{\text {a }}$ Padova, Italy
}

Recently, the feasibility and safety of percutaneous aortic valve replacement (PAVR) has been reported in the treatment of degenerative aortic valve stenosis in patients at high-risk for surgical aortic valve replacement (AVR). ${ }^{1}$ However, so far this therapy has been limited to patients with severe stenosis of the native valve. We report the case of a patient with severe aortic regurgitation owing to bioprosthesis dysfunction who was successfully treated by implantation of a CoreValve (CoreValve Inc, Irvine. Calif) prosthesis with a totally percutaneous approach.

\section{CLINICAL SUMMARY}

An 84-year-old woman, with previous surgical AVR with a bioprosthesis (Biocor $25 \mathrm{~mm}$ stentless; (Biocor Industria e Pesguisa Ltda, Belo Horizonte, Brazil) and cardiac pacemaker implantation for severe aortic stenosis in 1998, was admitted to a community hospital because of pulmonary edema. Comorbidity included hypertension, chronic renal failure, and previous left hemicolectomy for bowel malignancy. Transthoracic echocardiogram revealed a severe transprosthetic aortic regurgitation caused by leaflet degeneration and prolapse; the left ventricle was enlarged with moderate impairment of systolic function. Despite medical treatment, clinical status rapidly worsened. The case was discussed with two different surgical teams, who deemed the patient at high risk for redoing AVR because of advanced age, the risks of the redo procedure, and comorbidities: the logistic EuroScore was $31.8 \%$. Thus,

\footnotetext{
From the Interventional Cardiology, ${ }^{\mathrm{a}}$ Institute of Anaesthesiology, ${ }^{\mathrm{b}}$ and Institute of Cardiac Surgery, ${ }^{\mathrm{c}}$ Department of Cardiac Thoracic and Vascular Sciences, University of Padova, Italy.

Received for publication June 9, 2008; accepted for publication July 6, 2008; available ahead of print Dec 22, 2008.

Address for reprints: Massimo Napodano, MD, Interventional Cardiology, Department of Cardiac Thoracic and Vascular Sciences, University of Padova, 2 via Giustiniani.35100. Padova. Italy (E-mail: massimo.napodano@gmail.com).

J Thorac Cardiovasc Surg 2009;138:1027-8

$0022-5223 / \$ 36.00$

Copyright (c) 2009 by The American Association for Thoracic Surgery

doi:10.1016/j.jtcvs.2008.07.061
}

the patient was transferred to our department to evaluate the feasibility of PAVR. Cardiac catheterization and angiography confirmed the severity of aortic regurgitation with left ventricular dysfunction, increased ventricular filling pressure, pulmonary hypertension, and decreased cardiac index. The computed tomographic scan of the aorta and iliac and femoral arteries showed a moderate degree of wall calcification in the ascending aorta, with aortic root and annular dimensions amenable for PAVR; the femoral and iliac arteries showed a caliber suitable for large sheath insertion. The procedure was performed with the patient under mild sedation and local anesthesia by a percutaneous retrograde approach. Over an $18 \mathrm{~F}$ sheath percutaneously inserted in the right femoral artery, the valve (29 mm, third-generation CoreValve Revalving system) was introduced and retrogradely advanced under fluoroscopic guidance over a stiff wire in the ascending aorta across the pre-existing prosthesis plane. After careful evaluation of prosthesis position by angiography, the prosthesis was progressively deployed and the delivery system retrieved. Aortic angiogram after deployment showed the correct positioning of the prosthesis with a trivial paravalvular leak (Figure 1); no transvalvular gradient was detected. Finally, the $18 \mathrm{~F}$ sheath was removed and hemostasis of the right femoral artery was successfully obtained by knotting the sutures of a prepositioned suture-based closure device (Prostar XL 10F; Abbott Vascular, Alameda, Calif). The in-hospital course was uneventful and the patient was discharged at home on day 6 after the procedure. Dual antiplatelet treatment was prescribed for 6 months. At 6 months' follow-up, the patient remained free of adverse events, with persistent New York Heart Association functional class I; a transthoracic echocardiogram confirmed good performance of the implanted prosthesis with neither aortic regurgitation nor significant transprosthetic gradient.

\section{DISCUSSION}

Recently, Wenaweser and associates ${ }^{2}$ reported the first case of CoreValve implantation for the treatment of aortic regurgitation in a degenerating bioprosthesis. They used 